\title{
Non-linear jet-flap interactions: a dynamical-systems analysis
}

\author{
Onofrio Semeraro, Peter Jordan` Vincent Jaunetł and François Lusseyran\$
}

\begin{abstract}
We analyze the temporal dynamics associated with the jet-flap interactions by carryingout a dynamical-systems analysis. The experimental cases are characterized by three different set-ups of the jet-flap system, running in the range $M a=0.6-1.0$. The analysis is based on data presented by Jordan et al., ${ }^{1}$ where the self-sustained oscillations were analyzed by means of linear models. Nonlinear competition among the modes was observed: here we analyze this interplay by investigating the system using statistical tools, phase portraits, Poincaré sections, and return maps. We estimate the minimal number of degrees of freedom necessary for the description of a nonlinear model. The correlation dimension is assessed for four representative cases. Finally, we analyze the toroidal geometry in the phase-space and identify the main ingredients necessary for nonlinear reduced-order models of this system.
\end{abstract}

\section{Introduction}

Tonal dynamics occurring in an isothermal turbulent jet in presence of a sharp edge was recently analyzed by Jordan et al. ${ }^{1}$ The authors performed a thorough analysis of the tones as a function of the Mach number, the distance between the jet inlet and the edge position, and proposed a linear model based on stability analysis. It is suggested that the strongest tones are due to a coupling between the trapped modes (identified by the same team in Towne et al. ${ }^{2}$ and Schmidt et al. ${ }^{3}$ and existing in the range $0.82<M a<1$ ) and the Kelvin-Helmholtz wavepackets dominating the near-field dynamics of the turbulent jets. ${ }^{4,5}$ Predictive models as well as physical explanations of the feedback cycle induced by the presence of the flat plate in the jet flow are discussed therein. We refer the interested reader to the work by Jordan et al. ${ }^{1}$ for more details. Here, starting from the associated pressure measurements, we aim at deepen the analysis by qualitatively assessing the nonlinear dynamics of the underlying system. The analysis closely follows the one carried out by Lusseyran et al. ${ }^{6}$ The main goal of the contribution is to assess which kind of reduced-order models are appropriate for the description of the nonlinear dynamics and to provide a complementary perspective to the current modeling.

\section{A. Experiments and available measurements}

The setup of the experiment is shown in Fig. 1. The experiments were performed at the Bruit et Vent jetnoise wind tunnel of the Institut PPrime in Poitiers (FR). In the sketch, on the left, it is shown the round nozzle of diameter $D=0.05 \mathrm{~m}$, placed at a distance $L$ from the lower edge of the aluminium plate. The plate

\footnotetext{
${ }^{*}$ Research Scientist, LIMSI, CNRS, Université Paris-Saclay, 91400 Orsay, France

†Senior Research Scientist, Département Fluides, Thermique et Combustion, Institut Pprime, CNRS, Université de Poitiers, ENSMA, 86000 Poitiers, France

${ }^{\ddagger}$ Assistant Professor, Département Fluides, Thermique et Combustion, Institut Pprime, CNRS, Université de Poitiers, ENSMA, 86000 Poitiers, France

§Senior Research Scientist, LIMSI, CNRS, Université Paris-Saclay, 91400 Orsay, France

Copyright (c) 2019 by The Authors. Published by the American Institute of Aeronautics and Astronautics, Inc. with permission.
} 


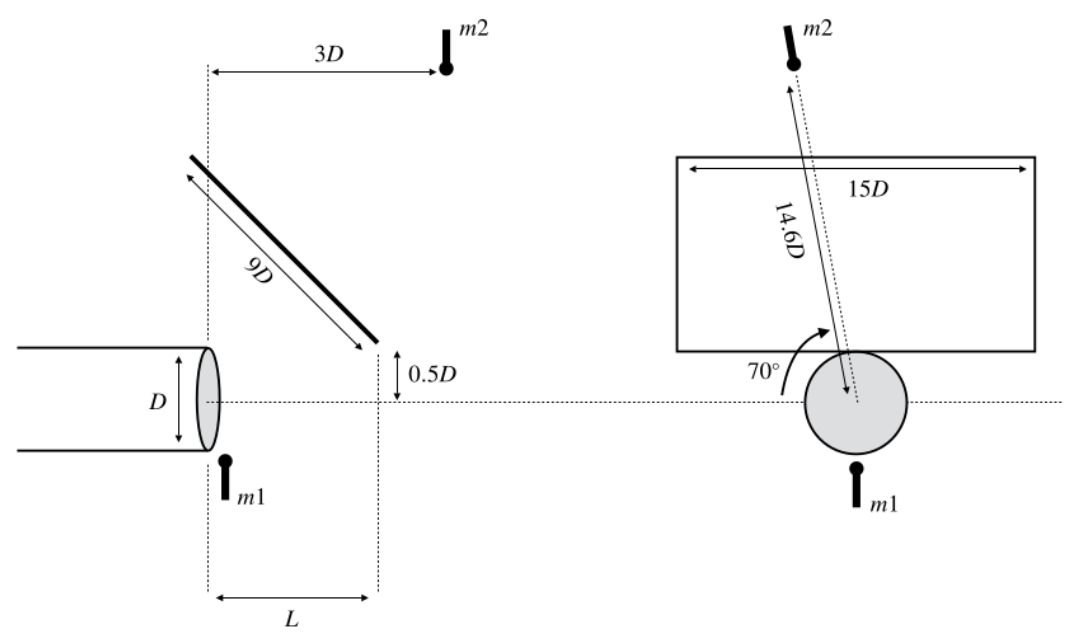

Figure 1. Scheme of the jet-flap interaction experiment. The figure is adapted from Jordan et al. ${ }^{1}$ A brief description of the setup is contained in Sec. IA.

is of dimensions $9 D \times 15 D \times 0.06 D$, as shown on the right side in Fig. 1, and inclined with respect of the streamwise direction of $45^{\circ}$. The boundary layer inside the nozzle is tripped by a strip placed $2.7 D$ upstream of the jet inlet (see for instance Cavalieri et al. ${ }^{4}$ and related works). The measurements are extracted from two microphones, recording the pressure fluctuations. The first microphone $\left(m_{1}\right)$ is close to the inlet of the jet, for capturing the pressure signature of the hydrodynamic near field at $(x / D, r / D)=(0.08,0.55)$; the second microphone $\left(m_{2}\right)$ is placed in the far field for the analysis of the acoustic field (the position is reported in Fig. 1). We synthesize in the following the main parameters of the available dataset of measurements.

- The Mach number is varied between $M a=0.6$ and $M a=1.0$, with increments of $\Delta M a=0.02$.

- The distance $L$ between the inlet and the edge of the plate is varied between $L=2 D$ and $L=4 D$ with increments of $1 D$.

- The sample rate of recording is $200 k H z$ for 20 seconds of acquisition time.

Hereafter, the frequency $f$ will be expressed using the Strouhal number defined as $S t=f D / U_{j}$, where $U_{j}$ is the velocity at the inlet and $f$ is in $H z$. Time will be scaled in nondimensional units as $t^{*}=t U_{j} / D$. In Fig. 2-4, on the left column, the power spectral densities (PSD) are shown as a function of the Ma the $S t$ for the configurations $L=[2 D, 3 D, 4 D]$. The signatures of the tonal modes are indicated by lighter colors.

\section{B. Aim of the present investigation}

In Fig. 5, the pressure fluctuations in time-domain, the spectrograms and the Fourier spectrum are shown for 4 different cases, the same as in Jordan et al. ${ }^{1}$ (Fig. 15, pag. 349 ibidem): case $A$ ), $M a=0.60, L / D=2$; case $B), M a=0.80, L / D=3$; case $C), M a=0.78, L / D=3$; case $D), M a=0.80, L / D=4$. In the figure, the PSD is computed by scaling the quantity by the frequency rate and taking the $\log _{10}$. We choose those cases as they are rather representative of the different dynamics were found in the jet-flap interaction. In particular, case $B$ is characterized by one frequency emerging in the spectrum, and corresponding to the dominance of a single limit cycle as it is possible to verify in the related phase-space. Cases $C$ and $D$, both at $L / D=4$, are characterized by the coexistence of two distinct peaks: those are the cases where the competition among the two modes can be found in the spectrogram. Finally, in case $A$, the co-existence of neighbour resonance cycles can be observed. In this work, the fluctuations in time will be defined as time series. The aim of this work is the statistical analysis of the time series dynamics. This analysis is the 
first step before a deeper analysis by means of system identification can be performed. ${ }^{7-9}$ The final goal is educing by data-driven approach features related to the non-linear interactions at play in this oscillator-type system. To a certain extent, the present analysis is the natural continuation of the work started by our team in Semeraro et al., ${ }^{10}$ where the temporal dynamics associated with the axisymmetric mode of an isothermal turbulent jet at $M a=0.6$ was taken under consideration (see also Breakey et al. ${ }^{11}$ ). However, it is worth to stress two substantial differences:

- The present flow exhibits an oscillator-type dynamics, which is more suitable for identification purposes

- We do not consider a restricted dynamics as in, ${ }^{10}$ but the global dynamics of the jet-plate interaction.

The paper is organized as follows. In Sec. II, we briefly summarizes some basic tools from time series analysis, with focus on the embedding and correlation dimensions, and present the results obtained for the present dataset of time series. In Sec. III, we focus on the dynamics at $M a=0.78, L / D=4$, where the spectral analysis suggests a competition in between two modes and the presence of intermittency; using the results obtained from a Poincaré section, we propose a possible, qualitative description of the nonlinear interactions. Some perspectives for future work are summarized together in the conclusions in Sec. IV.

\section{Dynamics from the observables: phase-space and dimensions}

\section{A. Embedding and correlation dimensions}

In principle, a dynamical system can be qualitatively analyzed by identifying the - unknown - underlying structure of the phase-space from a sequence of measurements of its state. Indeed, if the time series contains "enough information" from the system dynamics - i.e the system is observable from the measurements ${ }^{12}-$ we can define an embedding space, built using these measurements. ${ }^{13}$ From the geometric point of view, an embedding is a mapping which preserves the topological features; the embeddings are thus of interest for reconstructing the unknown phase-space, possibly with a minimal number of degrees of freedom which are meaningful for describing the dynamics. Not surprisingly, these concepts coming from signal processing and time-series analysis (see Kantz \& Schreiber $^{8}$ ) are now rediscovered within the field of deep learning for the analogies with neural networks embeddings. In that sense, any attempt of nonlinear reduced-order modeling based on time-series - as we will often stress in this work - needs to rely on a preliminary statistical analysis.

We state now a key results, the Takens' theorem, ${ }^{14}$ according to which the minimal dimension of embedding $(m)$ is linked to the correlation dimension $D_{2}$ as it follows

$$
m \leq 2 D_{2}+1 .
$$

More in details:

- The minimal embedding dimension $m$ is the minimal number of degrees of freedom required to describe the dynamics of the system;

- The correlation dimension $D_{2}$ is a measure of the dimension of an attractor in a phase space. ${ }^{15}$

The first step for the estimation of these quantities is the construction of an embedding space. As the time series $y(t)$ are typically univariate, an embedding matrix can be obtained using time-delay coordinates

$$
\mathbf{H}(m, \Delta t)=[y(T), y(T+\Delta t), y(T+2 \Delta t), \ldots, y(T+m \Delta t)],
$$

where $T$ indicates the time-span and $\Delta t$ the time-shift; the last row will have a total shift of $m \Delta t$. The singular values decomposition (SVD) of the Hankel matrix $\mathbf{H}$ in Eq. 2 is an alternative method for building an embedding space, where the associated left orthogonal modes correspond to the coordinates. A third possibility is the derivative embedding, where each coordinate is represented by the time-derivative of increasing 

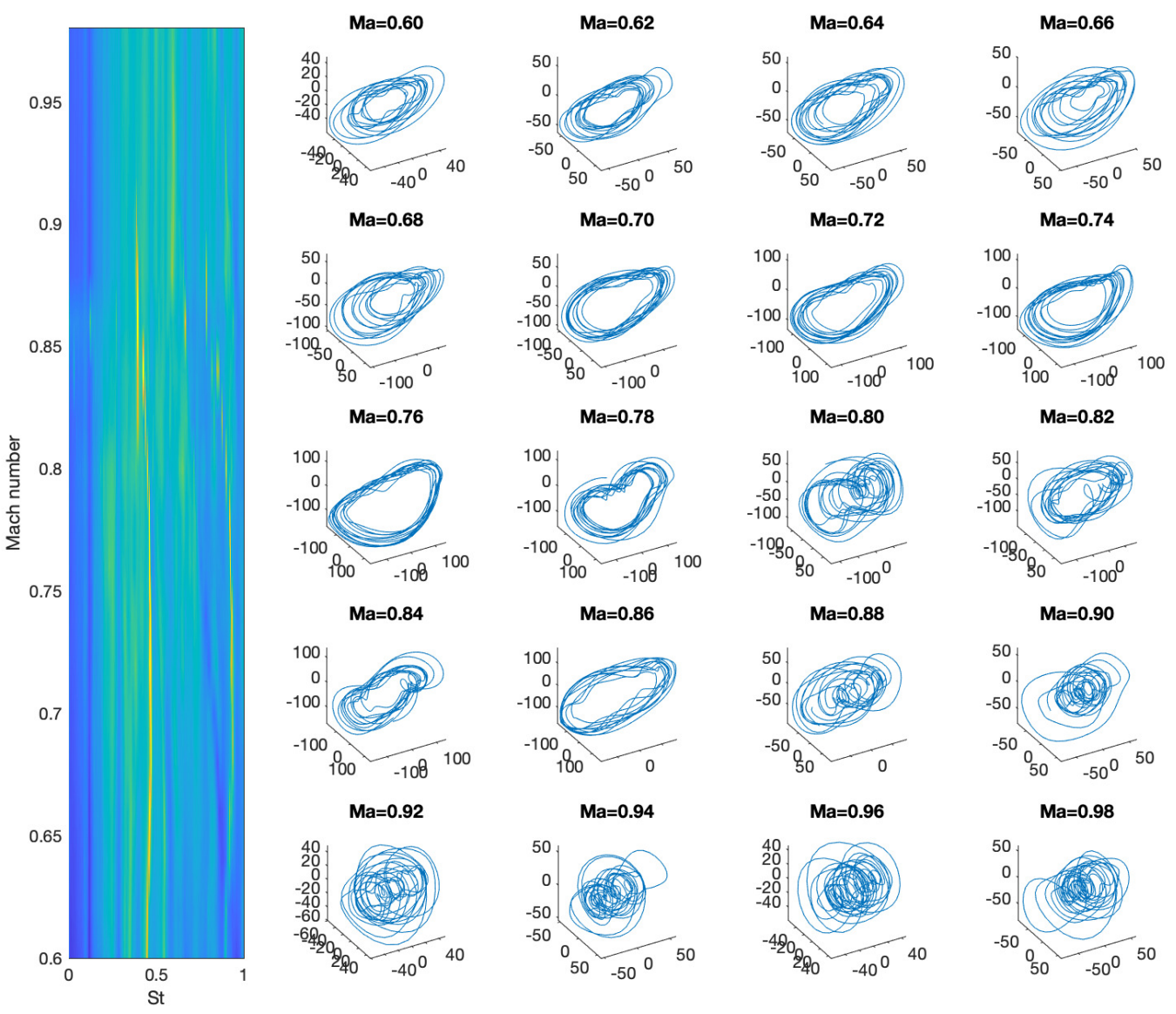

Figure 2. Left: spectral analysis performed by Welch's method showing the signatures of the most amplified frequencies as a function of the $S t$ and the $M a$. We consider here the configuration with $L=2 D$. Right: in the insets, obtained at different Mach numbers, it is shown the unfolded dynamics using time-delays. The time-shift for each coordinated is $\Delta t^{*}=0.14$, where $t^{*}$ is expressed in non-dimensional time units.

order of the time series. ${ }^{8}$ Although it is more delicate from the numerical point of view, the derivative embedding is interesting for system identification purposes: the resulting reconstructed state-space is in the form

$$
\begin{aligned}
\dot{y} & =f(y) \\
\dot{y_{1}} & =f\left(y_{1}, y\right) \\
& \ldots \\
\dot{y_{m}} & =f\left(y_{m}, y_{m-1} \ldots, y_{1}, y\right),
\end{aligned}
$$

which - in principle - allows to extract by system identification the equation of motion. For an interesting overview we refer to the work by Crutchfield, ${ }^{16}$ while applications along this line can be find for simple models in the works by Brunton et al. ${ }^{17,18}$ With this in mind, it is now easier to realize that the minimal embedding dimension is strongly related to the minimal number of equations that is necessary for describing the system underlying the measurements - if deterministic. In that sense, the system identification procedure 

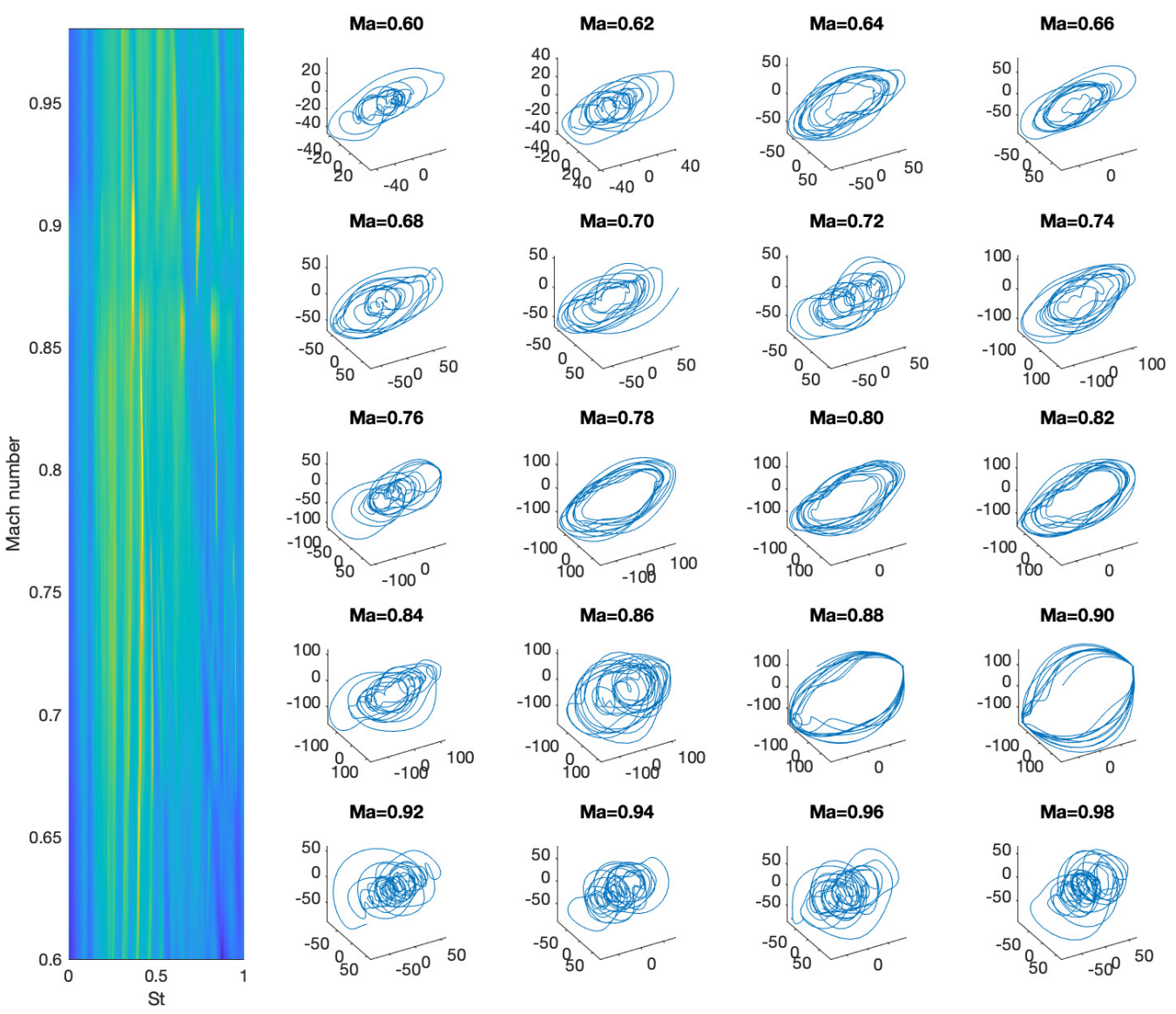

Figure 3. Left: spectral analysis performed by Welch's method for configuration with $L=3 D$. Right: in the insets at different Mach numbers, it is shown the unfolded dynamics using time-delays. The time-shift for each coordinated is $\Delta t^{*}=0.14$.

- whether one should use polynomial, Fourier or basis functions, as well as neural networks - can be only the second step of the modeling process once the statistical assessment is done.

When the subspace of projection is identified, the dynamics of the system can be evaluated. As already mentioned, according to the Takens' theorem and for the properties of the embedding, the geometrical properties of the original attractors are unchanged in the new subspace of projection. Thus, the correlation dimension $D_{2}$ can be estimated in the embedding space.

Before discussing the results for our cases, it is worthy to note that:

- Noise in the measurements typically increases the estimated embedding dimension. For experimental measurements, this is a strong limitation for the difficulties in separating what is related to noise measurements and what are the effects of the noise influencing the dynamics itself (see Cencini et al. $\left.{ }^{19}\right)$.

- Observability of the phenomena from the measured time series plays a fundamental role for extracting a correct model, as well as meaningful statistical dimensions and/or phase-space portraits. 

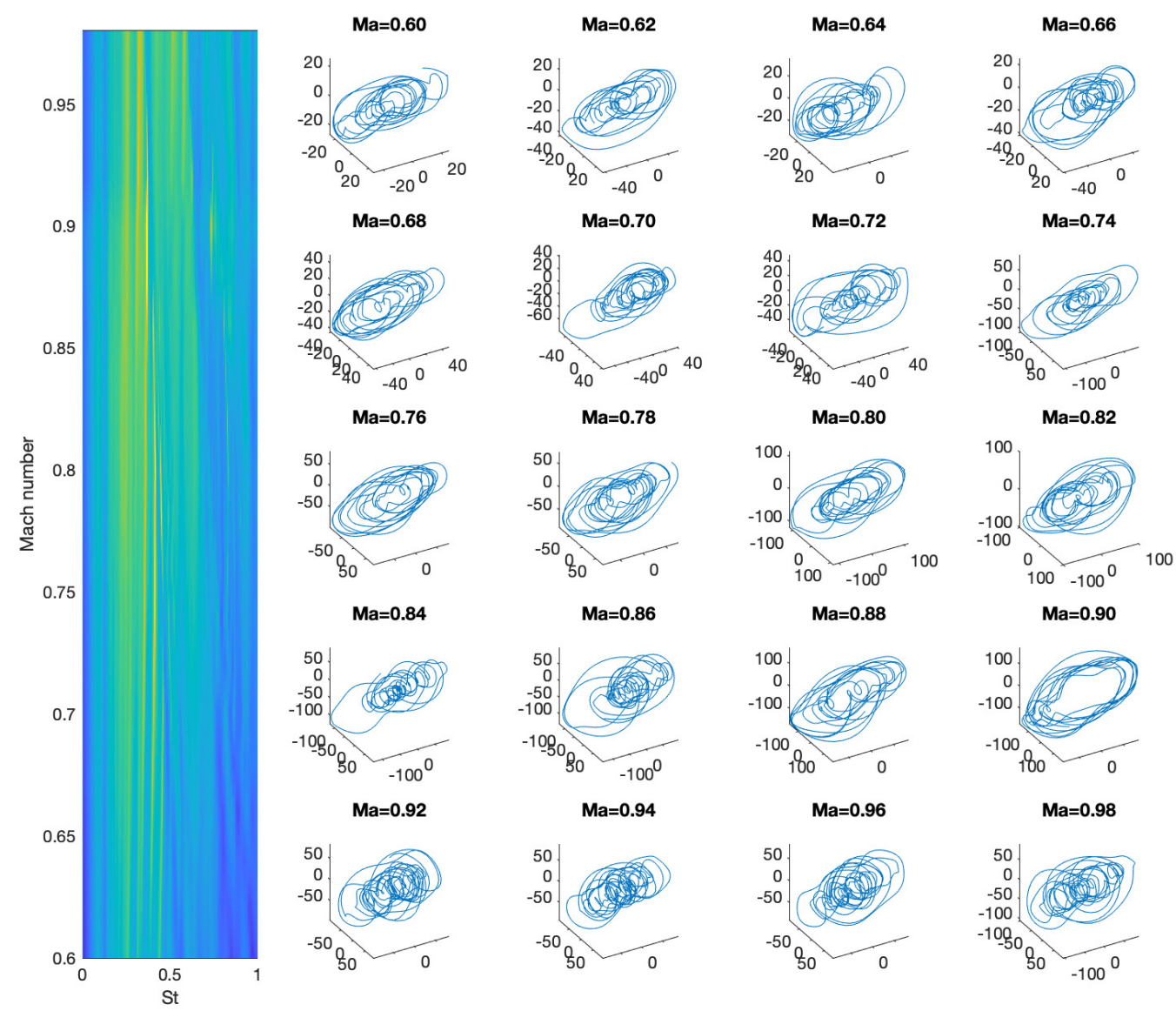

Figure 4. Left: spectral analysis performed by Welch's method for configuration with $L=4 D$. Right: in the insets at different Mach numbers, it is shown the unfolded dynamics using time-delays. The time-shift for each coordinated is $\Delta t^{*}=0.14$.

- The three methods of embedding are topologically equivalent.

In the following we will mainly use time-delayed and SVD embeddings; the time series are pre-processed with low-pass filtering, with cut-off frequency around $S t=3.25$. We do not apply any moving-average non-linear filtering, often used in similar studies (see Semeraro et al. ${ }^{10}$ ).

\section{B. Phase space portraits}

In Fig. 2-4, for each of the time series, we present the reconstructed phase-space. In the following we use the time-delayed embedding with a shift of $\Delta t^{*} \approx 0.14$. We take as an example the case $L / D=2$, in Fig. 2 : dominance of one frequency corresponds to a cleaner toroidal structure in the phase-space. However, this geometry is not related only to the presence of discrete frequencies, but it is also strongly influenced by the presence of the background turbulence as it will be further discussed in Sec. III. Co-existence of frequencies corresponds to more complex geometries of the phase-space. For $M a \rightarrow 1$ the scenario is less clear. Similar observations can be carried out also for the cases $L / D=3,4$, although the dynamics is progressively richer as the distance between the jet and the flat plate increases. 
$(A)$

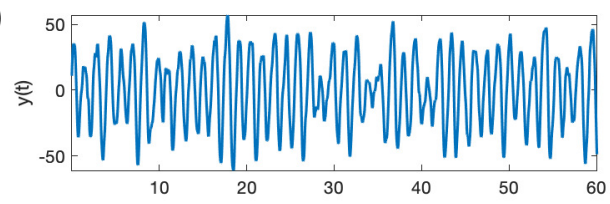

(B)

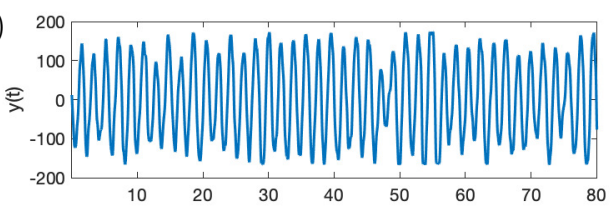

$(C)$

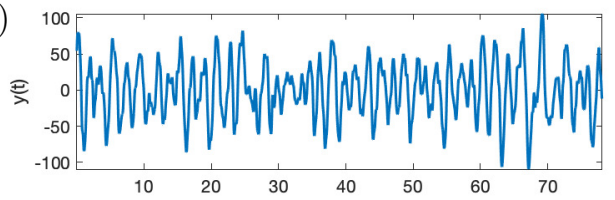

(D)

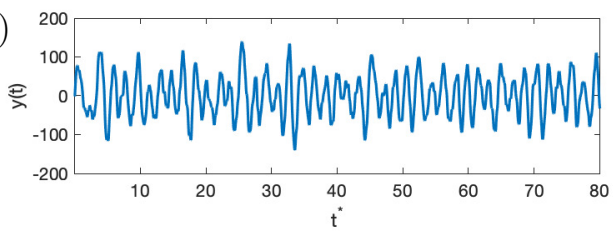

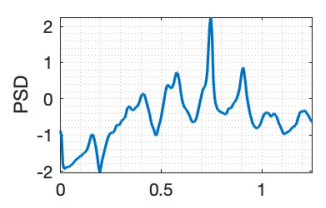
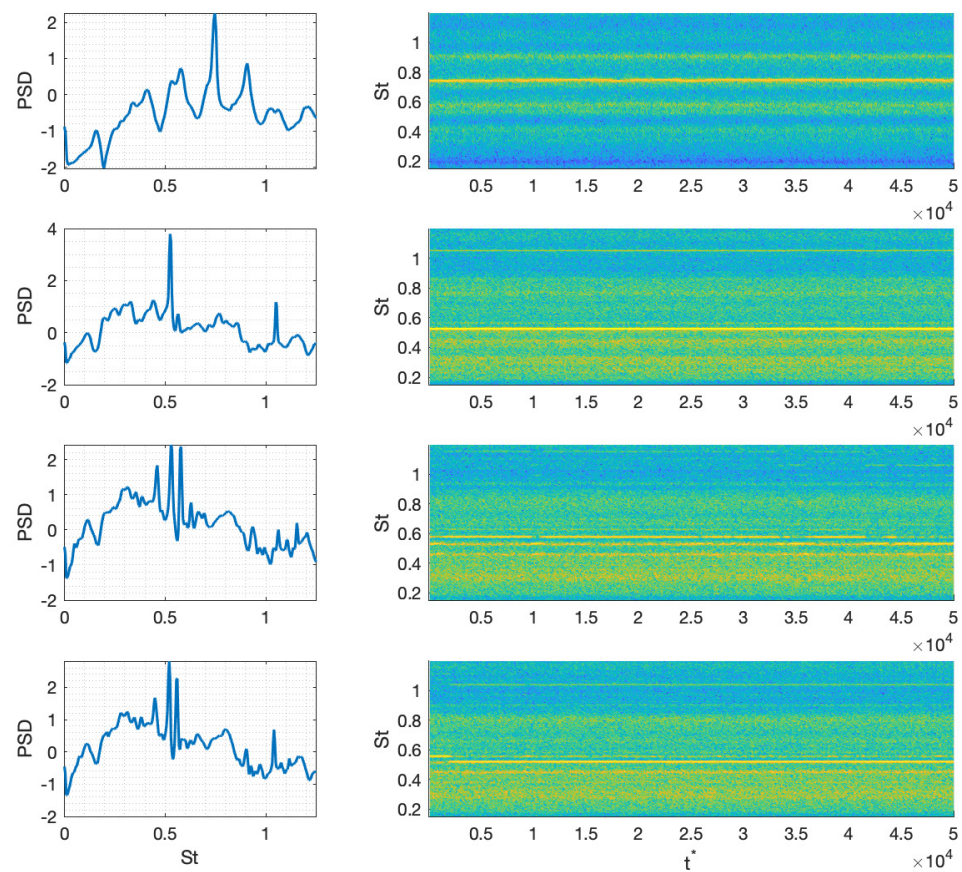

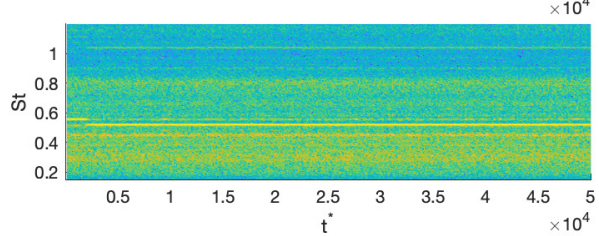

Figure 5. Pressure fluctuations as a function of time (left column), power spectral density (PSD) as a function of $S t$, obtained by means of a Welch analysis (center column) and spectrogram of the PSD as function of time $t^{*}$ and $S t$ for the reference cases: $(A) M a=0.60, L / D=2 ;(B) M a=0.80, L / D=3 ;(C) M a=0.78, L / D=4 ;(D)$ $M a=0.80, L / D=4$. A richer dynamics is observed for the cases $C-D$, characterized by $L / D=4$, due to the interaction among three distinct frequencies; in contrast, a simpler dynamics is obtained for $A$ and $B$.

\section{Embedding dimension}

The embedding dimension is computed by applying the algorithm described by Cao. ${ }^{20}$ The Cao function is defined as

$$
e(m)=\frac{1}{T-m \Delta t} \sum_{i=1}^{T-m \Delta t} a(i, m),
$$

where $T$ is the time-span of the time series, $\Delta t$ is the shift applied for the time-delay coordinates and $a$ is the parameter

$$
a(i, m)=\frac{\left\|y_{i}(m+1)-y_{n(i, m)}(m+1)\right\|}{\left\|y_{i}(m)-y_{n(i, m)}(m)\right\|}
$$

based on the time series in Eq. 2 with $i=[1,2, \ldots, T-m \Delta t]$ and $\|\cdot\|$ being a measure of the Euclidian distance. The subscript $n(i, m)$ indicates an integer value such that $y_{n(i, m)}$ is the nearest neighbour of $y_{i}$. The parameter $e_{1}$ is obtained as

$$
e_{1}(m)=\frac{e(m+1)}{e(m)}
$$

In practice, the value $e(m)$ in Eq. 3 is obtained as a statistical average performed over the channels of the embedding matrix $\boldsymbol{H}$. The algorithm is applied starting from lower values of $m$, that are increased by unitary steps until $e_{1} \rightarrow 1$. 

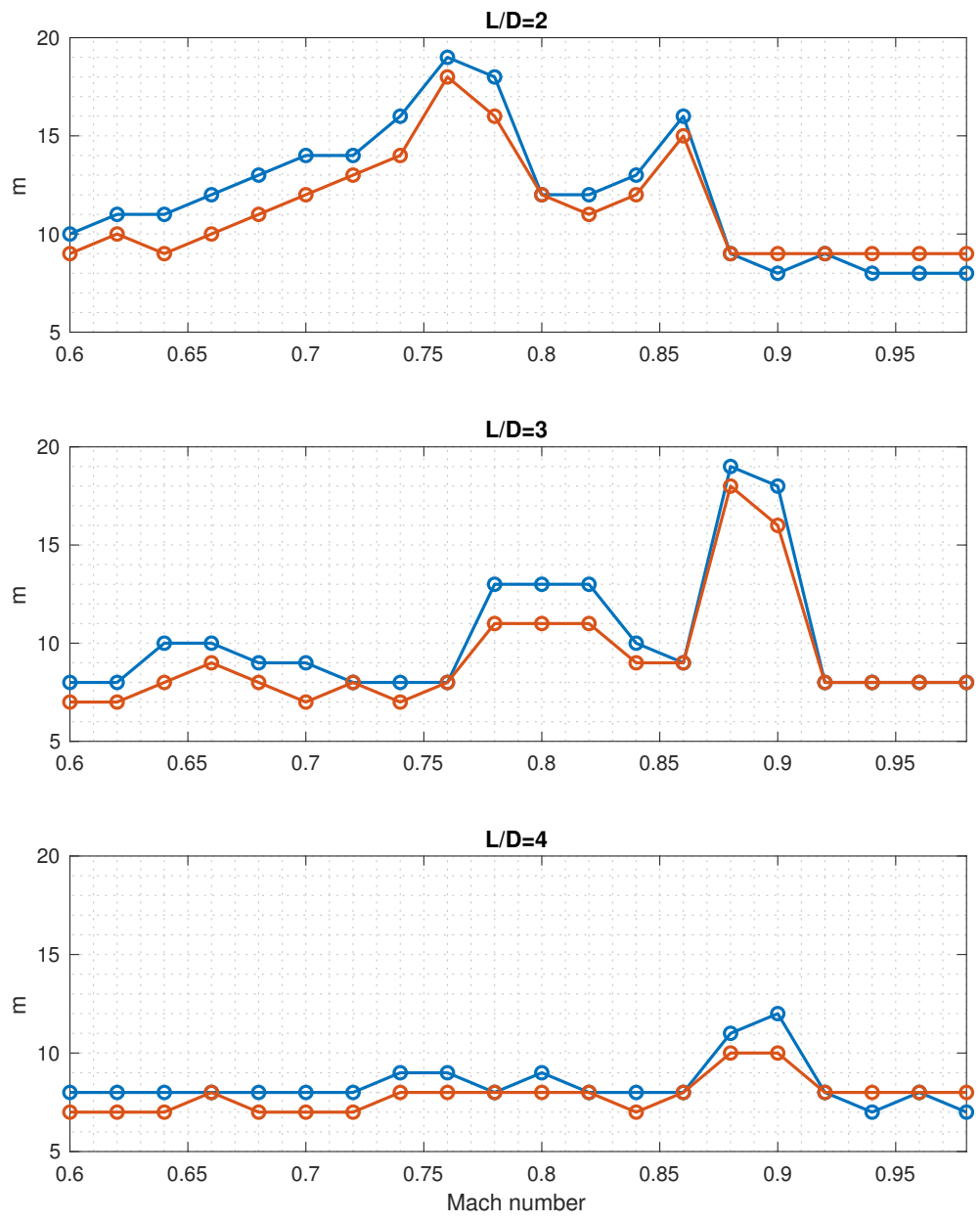

Figure 6. Minimum embedding dimensions obtained by applying the Cao's algorithm for all the cases $L / D=$ $2,3,4$, as a function of the Mach number. The blue lines indicate the results obtained by using different time delays: $\Delta t=10$ (case blue), $\Delta t=15$ (case orange).

Convergence Analysis For computing the dimension $m$ we compare 5 datasets of $N=40000$ points taken from each of the time series, corresponding to $t^{*} \approx 1070$; the initial point of each of these intervals is taken randomly. We verified that the results of each of the dataset randomly stacked from the time-series are consistent and that this amount of points is sufficient for guaranteeing convergence; for this reason, we can further average the results of the 5 sets. The maximum number of dimension explored is $M=25$ and we consider several $\Delta t^{*}$ of unfolding in the interval $\Delta t^{*}=\{0.14,0.27,0.40,0.53,0.67,0.80\}$. We found that an optimal choice for the convergence of the analysis is obtained for $\Delta t^{*}=\{0.27,0.40\}$. We took as a threshold for $e_{1}=0.99$.

RESUlts The results of the analysis are contained in Fig. 6. We consider two sets of data: the ones obtained with an unfolding $\Delta t^{*}=0.27$ (in blue) and with $\Delta t^{*}=0.40$ (in orange). The data in Fig. 6(a) are rather surprising when comparing the case $L / D=2$ with the cases $L / D=\{3-4\}$. In particular, for $L / D=2$, it can be observed that the dimension $m$ constantly grows from $m=9-10$ up to $m=18-19$ from $M a=0.6$ to $M a=0.75$, although the phase-space and the spectrum suggest a simpler dynamics in this interval of $M a$; for $M a>0.88$, where the harmonic is no longer so strong, the dimension is smaller and constantly equals $m=9$. In general, the embedding dimension becomes high whenever a harmonic frequency appears in the data, which suggests an increasing due to quadratic non-linearities. 
The situation is easier to discuss when considering $L / D=3,4$. In the first case, the embedding dimension is higher between $M a=0.86$ and $M a=0.92$ where the dynamics is found to be more complex in the phase space and first harmonics appear in the spectrum; in this interval the dimension is $15<m<19$. For $M a>0.92$ and $M a<0.76$, where the harmonic is no longer so strong/apparent, the embedding dimension is below 10 with both the unfolding $\Delta t^{*}$. Same behavior is found for $L / D=4$, where we found a rather constant value for the minimal embedding dimension, below $m<10$ for most of the $M a$, except in presence of the harmonics between $M a=0.86$ and $M a=0.92$. In Tab. 1, we summarize the estimated $m$ for the four cases we will further discuss in the next section by computing the correlation dimension $D_{2}$.

Some Remarks Despite the rather high values obtained for $L / D=2$, the results are consistent with the trend we would have expected by looking at the spectrum of the three cases. In that sense, we do not attribute these results to numerical artifacts: the increasing of the embedding dimensions as a function of $M a$ as well as the consistency between the two different sets of computations, obtained for $\Delta t^{*}=0.27$ and $\Delta^{*}=0.40$, lead us to the conclusion that the results are correct, but might be affected by noise. Noise in measurements, in fact, can lead to an increase of the $m$ estimation. For this reason, we are currently re-analyzing the dataset using filtered time-series; the nonlinear, moving-average filters are the same already applied in Semeraro et al. ${ }^{10}$ and contained in the packages implemented by Kantz \& Schreiber. ${ }^{8}$

\section{Correlation dimension}

The second dimension of interest is the correlation dimension $D_{2}$; by definition, the correlation dimension is a measure of the dimensionality of the space occupied by a set of points. Its utility stays in the possibility of determining the (possibly fractional) dimensions of an attractor in phase space. First, a correlation sum is defined as the fraction of all possible pairs of points $\left(y_{i}, y_{j}\right)$ closer than a given distance $\varepsilon$ as

$$
C(\varepsilon)=\frac{1}{N_{p}} \sum_{i=1}^{N} \sum_{j=i+1}^{N} \Theta\left(\varepsilon-\left\|y_{i}-y_{j}\right\|\right),
$$

where $\Theta$ is the Heaviside step function and $N_{p}=N(N-1) / 2$ is the number of pairs. The correlation sum $C(\varepsilon)$ contains only the pairs with a distance smaller than $\varepsilon$, which from the physical viewpoint represents the different scales of the system under scrutiny. The quantity $D_{2}$ is proportional to $C \propto \varepsilon^{D_{2}}$; thus, once $C$ is computed, the $D_{2}$ can be estimated. In what follows, we adopt as main tool the routines implemented by Kantz \& Schreiber, ${ }^{8}$ based on the Grassemberg-Procaccia algorithm. ${ }^{15}$

CONVERgEnCE ANALYsis A limit characterizes the computation of the correlation dimension: its value over a decade cannot exceed $D_{2}=2 \log _{10} N$, where $N$ is the number of points in the time series (Eckmann-Ruelle limit, $\left.{ }^{21}\right)$. So, fairly large datasets need to be used for avoiding results which are mere numerical artifacts. In our case, we considered two sets of $N=2 \times 10^{6}$ points for each of the 4 cases in Tab. 1; these sets guarantee a rather large saturation value for $D_{2}$.

We apply SVD-embedding, by forming the Hankel matrix in Eq. 2 with $m=25$ and $\Delta t^{*}=0.14$. The choice of the SVD-embedding is based on the ability of the SVD of separating the scales of the system, from the larger, most energetic ones (contained in the first modes) towards the finer, included in the higher-order modes. Moreover, higher modes typically contain the residual noise, detrimental for the statistical counting. Oversampling is avoided by introducing the so-called Theiler window, which limits the correlation between points during the pair-counting (see ${ }^{8,15}$ ); we apply $\Delta t^{*}=0.41$, after convergence checks.

Results In Fig. 7, we provide an example of $D_{2}$ estimation for the case A. Convergence is observed when:

- The channels tend to coalesce;

- A constant slope is obtained in the chart $\varepsilon-C(\varepsilon)$, corresponding to a plateau for $\varepsilon-D_{2}$. 

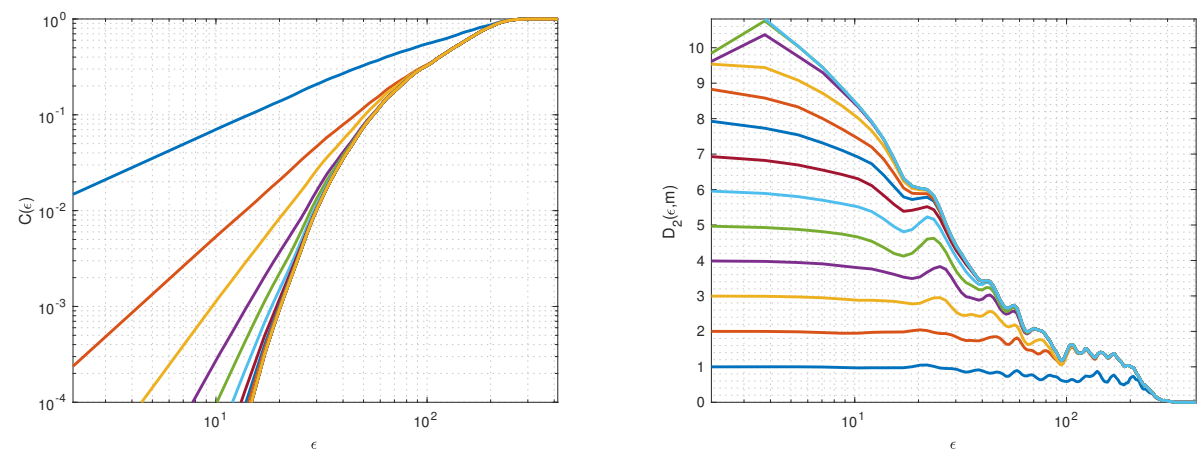

Figure 7. Case $M a=0.60, L / D=2$ : (left), correlation sum $C(\varepsilon)$ as a function of the analyzed scales $\varepsilon$; (right): correlation dimension $D_{2}$ as a function of the scales $\varepsilon$. Each line indicates a different channel ranging from $m=1$ (darker blue, first line from below) up to $m=13$. A plateau is observed at larger scales, roughly corresponding to $D_{2} \approx 1.4$.

\begin{tabular}{c|cc|ccc}
\hline \hline & Mach & $L / D$ & $m$ & $D_{2}$ & $m_{D_{2}}$ \\
\hline \hline Case A & 0.60 & 2 & $9-10$ & 1.4 & 4 \\
Case B & 0.80 & 3 & $11-13$ & $1.2-1.6$ & $4-5$ \\
Case C & 0.78 & 4 & 8 & $1.4-1.5$ & 4 \\
Case D & 0.80 & 4 & $8-9$ & $1.4-1.8$ & $4-5$ \\
\hline \hline
\end{tabular}

Table 1. Embedding $(m)$ and correlation $\left(D_{2}\right)$ dimensions for four cases. In the last column, $m_{D_{2}}$ provides an estimate for $m$ based on $D_{2}$ and the Takens' theorem

By comparing Fig. $7(a)$ and Fig. $7(b)$, we observe a plateau at larger scales $\left(\varepsilon>10^{2}\right)$ where eye inspection allows to get a value for $D_{2} \approx 1.4$. At smaller scales it is not possible to observe convergence; although it can be found for some scale-intervals the coalescence of the curves (each of them corresponding to a different channel), we cannot extract a value for $D_{2}$.

We summarize the obtained results in Tab. 1 for the reference cases. We consider the minimum and maximum value of $D_{2}$ where the plateau appears. In particular, the $D_{2}$ dimensions ranges from the "simpler" case in $B$, where we estimate a value in the range $D_{2}=1.2-1.6$ to more complex cases such as $C$ and $D$, where the maximum estimate is $D_{2}=1.8$. Moreover, the values never exceeds $D_{2} \approx 5$; from a practical point of view, a meaningful analysis of the nonlinear dynamics, based on time-series, can be only performed when the dimensionality of the system is relatively small. ${ }^{22}$

SOME REMARKS The values are rather consistent with the dynamics observed in the spectrum where discrete frequencies are found and the phase-space where most of the cases show the trajectories to be bounded by toroidal surface. These surfaces are characterized by one dominant frequency when the structure resembles a limit-cycle; on the other hand, in presence of more than one frequency, the presence of multiple frequencies lead the entire geometry to be closer to a torus ${ }^{23,24}$. All the analyzed cases, however, are characterized by stochastic forcing, whose implications are discussed in the next section.

We can also notice that the Takens' theorem is not respected, as the embedding dimension is, in principle, over-estimated in all the cases; in the last column of Tab. 1, we indicate an estimation of $m$ based on the correlation dimension. The discrepancy between $m$ and $m_{D_{2}}$ can be related to the presence of noise in the measurements that, despite the low-pass filtering, can affect the statistical counting of the minimal embedding dimension. Yet, a question raises rather naturally: is the $D_{2}$ dimension under-estimated for the choice of the scales $\varepsilon$ or the $m$ over-estimated for the presence of noise? We are currently reviewing these results by in-house codes for further validating the present results. 


\section{Nonlinear analysis: identifying intermittent cases from measurements}

The dimension analysis can be completed by directly perusing the dynamics observed from the time series. This can be done, when dimensions are small enough - as the results of Sec. II suggest - by applying a Poincaré map. By definition, a Poincaré map or first return map is the intersection of a trajectory orbit in the phase space with a lower-dimensional subspace, transversal to the "flow" of the system. In our case, the section is given by a 2-D plane crossing the 3-D attractor: the ensemble of points obtained at each loop provides a recurrence map. Thus, a recurrence map for a periodic-orbit or limit-cycle corresponds to one point; a torus with two frequencies whose ratio is a rational number provides an ensemble of points, while a torus with two frequencies, of which ratio is an irrational number is characterized by a continuous, closed line. Fractal geometries characterize strange attractors. For more details we refer to the specialized literature $^{23}$.

\section{A. Poincaré section and statistical counting}

In the following we focus on case $C$. The analysis closely follows the one performed in Lusseyran et al., ${ }^{6}$ where the application is the bimodal intermittency in an experimental cavity flow. In Fig. 8, all the steps from the Poincaré section to the angular map are exemplified. First, we consider a section (Fig. 8a). We consider points taken in a plane parallel to $(x, y)$, with $z=0$ and $\dot{z}>0$; the coordinates are obtained by time-delays of $\Delta t^{*}=0.27$. The red dots are the ones belonging to the Poincaré section. In Fig. $8 b$, a differential return map is shown, where the coordinates are $\left(y_{n+1}-y_{n}\right)$ versus $\left(y_{n+2}-y_{n+1}\right)$. The choices we made do not influence the results: sections including $x=0, y=0$ or oriented with the negative gradient, as well as the return maps based on the $x$ coordinate or the module $\sqrt{\left(x^{2}+y^{2}\right)}$ allow us to get equivalent results.

The section appears as a cloud of points. This geometry suggests a toroidal phase-space already destabilized, where all the points delimited by a toroidal surface are visited during the trajectory. From the spectrum in Fig. 9, we can observe the presence of two main modes: $f_{1}$ at $S t=0.577$ and $f_{2}$ at $S t=0.532$; the spectrum shows a third peak at $S t=0.460$, with a lower amplitude. The return map does not allow an easy distinction between the deterministic nonlinear dynamics and the stochastic process, contaminating the dynamics itself; moreover, the spectral analysis suggests competition of the two distinct modes $f_{1}$ and $f_{2}$ (see also discussion in Jordan et al., ${ }^{1}$ ).

In Fig. $8 b$, we indicate with a red dot the baricentrum of the points' distribution. An angle $\theta_{n}$ can be computed for each point of the cloud in the differential map with respect of this baricentrum. Each angle is associated with the $n$-iterate, crossing the section, and is computed in counterclockwise direction, starting from the horizontal segment indicated in red in the picture. In Fig. $8 c, \theta_{n+1}$ is plotted versus $\theta_{n}$; the density function associated with the angles $\theta$ is shown in Fig. $8 d$ : it is possible to note a bimodal distribution obtained at different angles. In principle, the peaks in correspondence with the bisecting line in $(c)$, such that $\theta_{n+1} \approx \theta_{n}$, represent trajectories passing in the vicinity of a periodic orbit; the counting of samples along each of this periodic orbit allows a direct estimation of the frequency as $f=f_{s} / T$, with $f_{s}$ the sampling frequency.

However, this is not exactly the case in Fig. $8 c-d$ : despite a rather neat bimodal distribution, we do not observe a dense distribution of points along the bisecting line, but along the parallel lines. For explaining this discrepancy, we have counter-verified the procedure by modeling a two-dimensional torus; as already mentioned torus is typically characterized by two frequencies: one slow and one fast, in a ratio that can be a rational number (commensurate) or an irrational number (incommensurate). We verified that the procedure suggested in ${ }^{6}$ and used here is capable of detecting the fast frequencies, but not the slow ones, unless making use of ad hoc Poincaré sections or return maps of higher order; moreover, the tipical circle map of a torus is of the type shown $\mathrm{in}^{23}$ (chapter 6.2, pag. 225 for the $2^{\text {nd }}$ ed.), with the points of the Poincaré section approximately aligning along the lines parallel to the bisecting line.

Coming back to our case, in Fig. 10(a) - (b) the rather neat distribution of the angles $\theta$ allow to estimate the orbiting period of each loop, obtained by counting the samples along each trajectory. By selecting the 


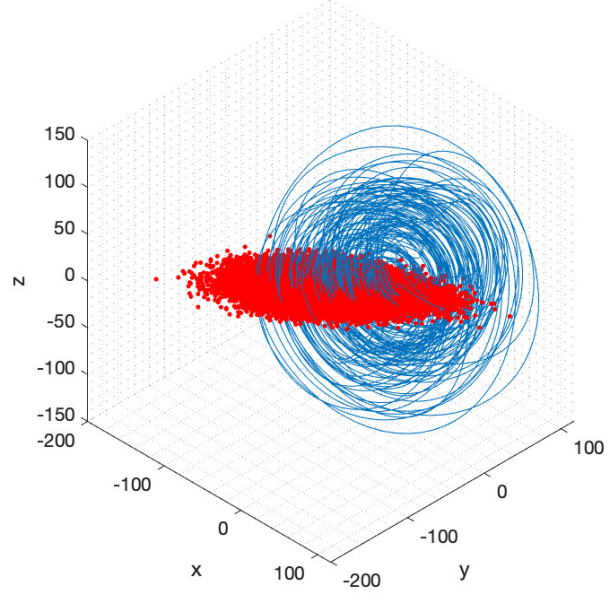

(a) Poincaré map

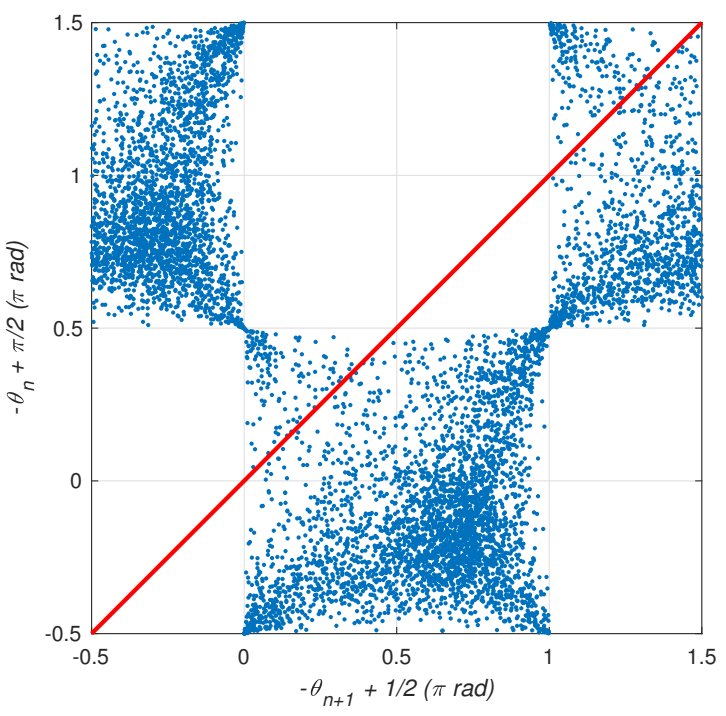

(c) Angular map

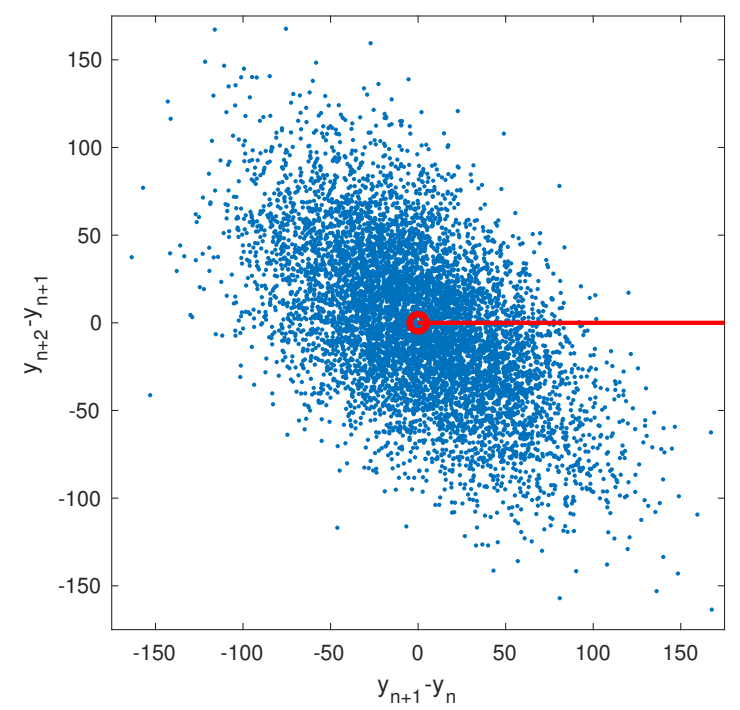

(b) Differential map

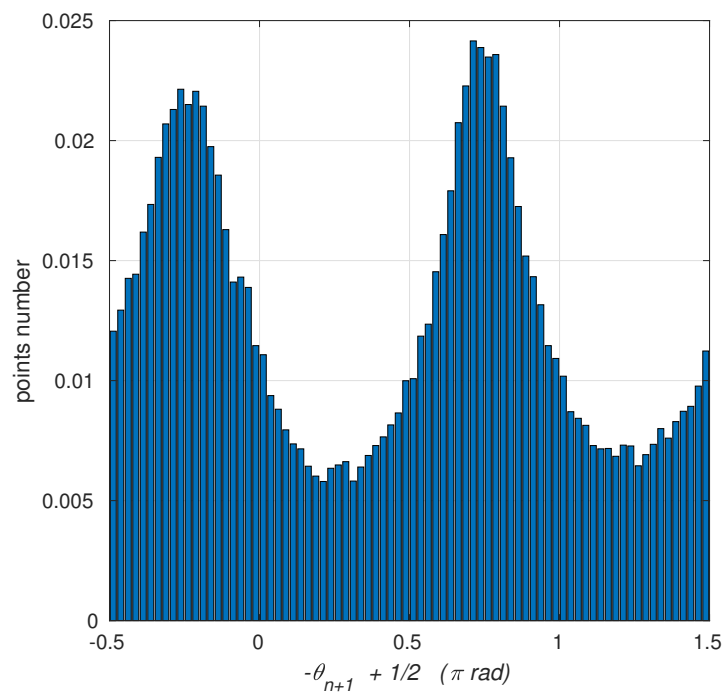

(d) Histogram, $\theta$ distribution

Figure 8. Statistical analysis. A Poincaré section is performed (a) by taking $z=0$ and $\dot{z}>0$. The points belonging to the Poincaré section are indicated with red dots. Using the points in the crossing plane, a first-return differential map of the coordinates $y_{n+2}-y_{n+1}$ vs $y_{n+1}-y_{n}$ is built. The red dot indicates the baricentrum of these points. With respect of the red dot and for each of the blue plot, the angle $\theta$ is computed in counterclockwise direction. The resulting angular map is shown in $(c)$, from which an histogram of the number of entries over the entire trajectory is obtained $(d)$. Note that the points are not as dense as expected along the bisecting line, but rather two parallels. 


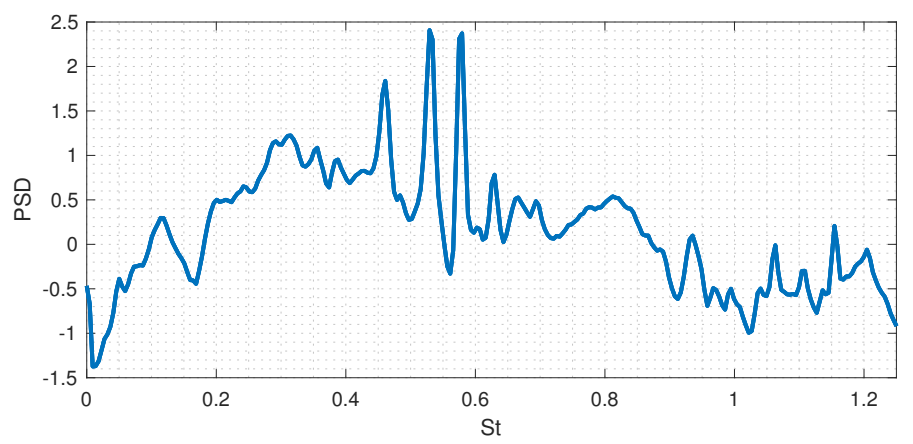

Figure 9. Welch analysis for $M a=0.78$ and $L / D=4$, same as Fig. 5, magnified for comparison with the analysis in Fig. 8-10.

\begin{tabular}{c|c|cc|cc}
\hline \hline & Fourier & Hist $\left._{\max }\right|_{1}$ & Ker $\left._{\max }\right|_{1}$ & Hist $\left._{\max }\right|_{2}$ & Ker $\left._{\max }\right|_{2}$ \\
\hline \hline$f_{1}$ & 0.5773 & 0.5701 & 0.5949 & 0.5549 & 0.5712 \\
$f_{2}$ & 0.5316 & 0.5238 & 0.5338 & 0.5299 & 0.5378 \\
\hline \hline
\end{tabular}

Table 2. Resulting frequencies: comparison between Fourier analysis and statistical counting

maximum we get as estimates of the frequency $S t_{f_{1}}=0.570$ and $S t_{f_{2}}=0.524$; by using a kernel fitting distribution (red curves in the Fig. 10), we get similar results: $S t_{f_{1}}=0.595$ and $S t_{f_{2}}=0.533$. These values are an accurate estimate of the frequencies educed by Fourier analysis (see Tab. 2). The density functions show also the presence of a second hump roughly at $T \approx 4.25-4.5$, i.e. $S t \approx 0.3$, so not corresponding to $S t_{f_{3}} \approx 0.44$.

A second estimation of the period is obtained by counting the number of switches between the two distributions. This is performed by using binary coding and boolean variables with the aim of separating the transitions between modes $\left(f_{1} \rightarrow f_{2}\right.$ vs $\left.f_{2} \rightarrow f_{1}\right)$ from orbits "staying" more than one loop. The results are listed in Tab. 2, with respect of the histogram diagram in Fig. $10 c-d$. When the kernel fitting is applied, we can observe a very good approximation of the original frequencies.

\section{B. Some remarks: why a statistical analysis using Poincaré maps?}

We can conclude that:

- We are capable at approximate the competing frequencies $f_{1}$ and $f_{2}$ from statistical counting, as demonstrated by the good approximation of the frequencies.

- We do not observe a distribution of the points in the angular map such that $\theta_{n} \approx \theta_{n+1}$, but along lines parallel to the bisecting line.

- The observed dynamics is compatible with intermittency between the main frequencies $f_{1}$ and $f_{2}$; a slow dynamics due to $f_{3}$ is not (and can not be) captured by this analysis.

- Considering the denser distribution along the parallels of the bisecting line in Fig. $8 c$, closely resembling what is seen in torii, we can conclude that the toroidal aspect of the map is due to the frequency $f_{3}-$ which makes the attractor "floating" in section of Fig. $8 a-$ in combination with the stochastic noise. Thus, the slow frequency is responsible of the toroidal surface, while stochastic forcing contributes at filling the entire phase-space. Scattered, outer, points can be seen as burst events. 


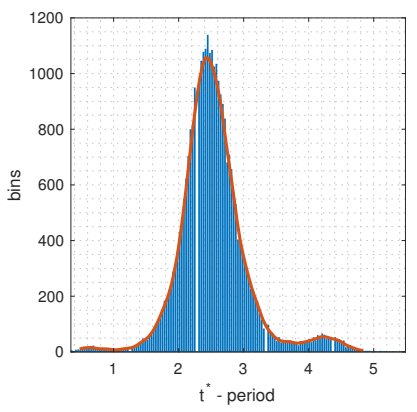

(a) Mode $f_{1}$

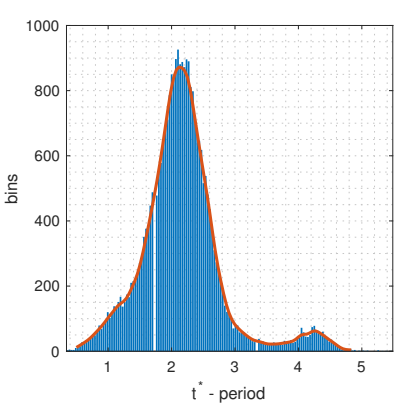

(b) Mode $f_{2}$

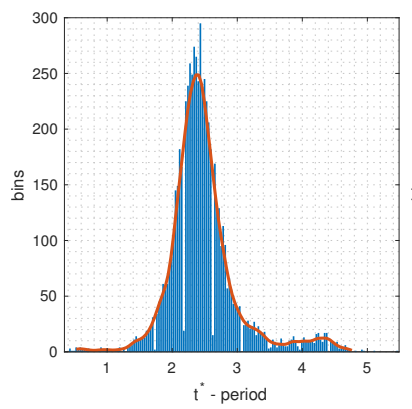

(c) Mode $f_{1}$

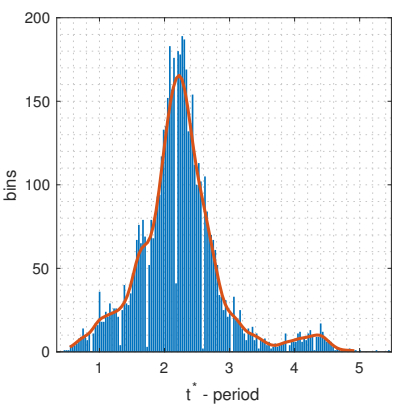

(d) Mode $f_{2}$

Figure 10. Density functions for the distribution of the periods for the modes $f_{1}$ and $f_{2}:$ i) $a-b$ direct counting (see text and Fig. $8 d$ ); ii) $c-d$ selection of the trajectories. The blue bars indicate the density function; the red lines for each of the distributions are obtained by a kernel fitting.

These considerations are of basic importance for the modeling, as we can build a skeleton based on these "ingredients" for a reduced order model of the dynamics.

The same analysis was applied in the other cases. In particular, in case $D$ we observe a similar separation for the dominating fast modes, although the frequency estimation was less acccurate if compared with $C$. This can be attribute to the slower switching observed by Jordan et al. in ${ }^{1}$ and thus to a less accurate statistical counting. For the case $A$ and $B$ instead, the analysis is not meaningful: the fast mode is correctly captured, while the slower modes responsible of the toroidal distribution in the phase-space are not captured by the current analysis, unless testing progressively higher return-maps which cannot reveal the fast modes.

\section{Conclusions}

We investigated the dynamics of the interactions between a turbulent jet and a flap: starting from the measurements of Jordan et al. ${ }^{1}$ we used tools from time series analysis and a Poincaré section for analyzing into the nonlinear dynamics of the system. We can summarize as follows our results

- We calculated the embedding dimension for all the cases, varying the $M a$ and the distance $L / D$. For the cases $L / D=\{3-4\}$ we found a dimension $m \approx 10$ except when more complex dynamics emerges. We found rather high values, unexpectedly, for $L / D=2$.

- The $D_{2}$ dimensions is calculated for 4 different cases, ranging from a resonant cycle to the slow/fast mode-switching. We found rather small values, in the range $1.2-1.8$, in contradiction with the estimated minimal embedding dimensions, but more consistent from a physical viewpoint.

- Finally, we borrowed tools from nonlinear dynamical systems theory for analyzing the mode competition of the case $M a=0.78, L / D=4$. By building the differential angular first-return map, we analyzed the Poincaré map obtained from the reconstructed phase-space. Statistical counting provides a rather good approximation for the frequencies of the two switching modes. Moreover, the angular map suggests that the toroidal surface is due to the appearance of a slow harmonic frequency. The stochastic processes fill the phase-space delimited by the toroidal surface. Outer points in the map can be attributed to burst events.

By combining these three analysis, we have a cleaner picture for the intermittent case, that allows us to have all the ingredients for building a nonlinear reduced-order model. Thus, future extensions of this work will be devoted to the system identification of nonlinear models $\left(\mathrm{see}^{7,9,17,18}\right)$. More in details, system identification can be of help in characterizing the nonlinear interactions among the modes and the stochastic forcing, in order to correctly reproducing the features observed in the experiments. This is a challenging task, as it is 
never obvious to properly separate the contribution of the stochastic behavior from the nonlinear interaction of the deterministic part of the model. In that sense, black-box modeling based on machine-learning strategies such as neural networks based on the sole reproduction of the temporal dynamics do not provide any deeper understanding of the system. For this reason, we do believe that only after an in-depth analysis as the one performed here it is possible to perform a physical-meaningful modeling of the dynamics of such a system.

Finally, we believe that the dimensions $m$ and $D_{2}$ deserve further verifications. We are performing again these computation on filtered time series (see examples in $^{8}$ or $^{10}$ ) for both the embedding dimension $m$ and the $D_{2}$ dimensions by applying in-house algorithm recently implemented from our group. As already mentioned, the importance of the minimal embedding dimension is closely related to an adequate reducedorder modeling.

\section{References}

${ }^{1}$ Jordan, P., Jaunet, V., Towne, A., Cavalieri, A. V., Colonius, T., Schmidt, O., and Agarwal, A., "Jet-flap interaction tones," J. Fluid Mech., Vol. 853, 2018, pp. 333-358.

${ }^{2}$ Towne, A., Cavalieri, A. V., Jordan, P., Colonius, T., Schmidt, O., Jaunet, V., and Brès, G. A., "Acoustic resonance in the potential core of subsonic jets," J. Fluid Mech., Vol. 825, 2017, pp. 1113-1152.

${ }^{3}$ Schmidt, O. T., Towne, A., Colonius, T., Cavalieri, A. V., Jordan, P., and Brès, G. A., "Wavepackets and trapped acoustic modes in a turbulent jet: coherent structure eduction and global stability," J. Fluid Mech., Vol. 825, 2017, pp. 1153-1181.

${ }^{4}$ Cavalieri, A. V. G., Rodríguez, D., Jordan, P., Colonius, T., and Gervais, Y., "Wavepackets in the velocity field of turbulent jets," J. Fluid Mech., Vol. 730, 2013, pp. 559-592.

${ }^{5}$ Jordan, P. and Colonius, T., "Wave packets and turbulent jet noise," Annu. Rev. Fluid Mech., Vol. 45, 2013, pp. 173-195.

${ }^{6}$ Lusseyran, F., Pastur, L., and Letellier, C., "Dynamical analysis of an intermittency in an open cavity flow," Phys. Fluids (1994-present), Vol. 20, No. 11, 2008, pp. 114101.

${ }^{7}$ Ljung, L., System identification, Wiley Online Library, 1999.

${ }^{8}$ Kantz, H. and Schreiber, T., Nonlinear time series analysis, Vol. 7, Cambridge university press, 2004.

${ }^{9}$ Billings, S. A., Nonlinear system identification: NARMAX methods in the time, frequency, and spatio-temporal domains, John Wiley \& Sons, 2013.

${ }^{10}$ Semeraro, O., Lusseyran, F., Pastur, L., and Jordan, P., "Qualitative dynamics of wave packets in turbulent jets," Phys. Rev. Fluids, Vol. 2, No. 9, 2017, pp. 094605.

${ }^{11}$ Breakey, D. E., Jordan, P., Cavalieri, A. V. G., and Léon, O., "Near-field wavepackets and the far-field sound of a subsonic jet," 19th AIAA/CEAS Aeroacoustics Conference, AIAA Paper, 2013, p. 2083.

${ }^{12}$ Aguirre, L. A. and Letellier, C., "Investigating observability properties from data in nonlinear dynamics," Physical Review E, Vol. 83, No. 6, 2011, pp. 066209.

${ }^{13}$ Carroll, T. L., "Testing Dynamical System Variables for Reconstruction," arXiv preprint arXiv:1808.08897, 2018.

${ }^{14}$ Takens, F., "Detecting strange attractors in turbulence," Dynamical systems and turbulence, Warwick 1980, Springer, 1981, pp. 366-381.

${ }^{15}$ Grassberger, P. and Procaccia, I., "Characterization of strange attractors," Physical review letters, Vol. 50, No. 5, 1983, pp. 346 .

${ }^{16}$ Crutchfield, J. P., "Between order and chaos," Nature Physics, Vol. 8, No. 1, 2012, pp. 17.

${ }^{17}$ Brunton, S. L., Proctor, J. L. P., and Kutz, J. N., "Discovering governing equations from data by sparse identification of nonlinear dynamical systems," Proceedings of the National Academy of Sciences, Vol. 113, No. 15, 2016, pp. $3932-3937$.

${ }^{18}$ Brunton, S. L., Brunton, B. W., Proctor, J. L., Kaiser, E., and Kutz, J. N., "Chaos as an intermittently forced linear system," Nature Communications, Vol. 8, 2017.

${ }^{19}$ Cencini, M., Falcioni, M., Olbrich, E., Kantz, H., and Vulpiani, A., "Chaos or noise: Difficulties of a distinction," Physical Review E, Vol. 62, No. 1, 2000, pp. 427.

${ }^{20} \mathrm{Cao}$, L., "Practical method for determining the minimum embedding dimension of a scalar time series," Physica D: Nonlinear Phenomena, Vol. 110, No. 1, 1997, pp. 43-50.

${ }^{21}$ Eckmann, J.-P. and Ruelle, D., "Fundamental limitations for estimating dimensions and Lyapunov exponents in dynamical systems," Physica D: Nonlinear Phenomena, Vol. 56, No. 2-3, 1992, pp. 185-187.

${ }^{22}$ Cencini, M., Cecconi, F., and Vulpiani, A., Chaos: from simple models to complex systems, Vol. 17, World Scientific, 2010.

${ }^{23}$ Ott, E., Chaos in dynamical systems, Cambridge University Press, 2002.

${ }^{24}$ Strogatz, S. H., Nonlinear dynamics and chaos: with applications to physics, biology, chemistry, and engineering, Westview press, 2014. 